\title{
A Hybrid Model for Tumor Radiotherapy Based on Kinetic Theory and Gene-Environment Network
}

\author{
Jinpeng Qi, Quan Gu*, Tao Han \\ College of Information Science and Technology, Donghua University, Shanghai, China. \\ * Corresponding author. Tel.: +86-21-67792301; email: supergu0925@163.com \\ Manuscript submitted October 1, 2016; accepted December 16, 2016. \\ doi: 10.17706/ijbbb.2017.7.1.51-57
}

\begin{abstract}
In this work, an optimal framework for tumor radiotherapy is investigated based on Kinetic Theory of Active Particle (KTAP) and Gene-Environment Network (GEN) model. The inner mechanism and the dynamic kinetics of radiotherapy are presented through complicated mutual interactions among molecular particles and acute Ion Radiation (IR) cofactors, including stochastic DNA damage generation, repair, and switch-like Ataxia Telangiectasia Mutated (ATM) activation, periodical oscillation of P53 network, as well as alternative phase of cell cycle arrest and apoptosis. The simulations can illustrate the outcomes of radiotherapy under different IR circumstances, including tumor cell mass degradation, toxin accumulation, and cell activity during the first and second phase of radiotherapy. Especially, an optimal strategy of radiotherapy is analyzed under different strength of IR circumstances.
\end{abstract}

Key words: Radiotherapy, optimal strategy, modeling, kinetic theory of active particle, gene-environment network.

\section{Introduction}

Cancer, like many other serious diseases, results from a complex interplay of genetic and environmental risk factors [1], [2]. As one kind of the main tumor therapies, radiotherapy is a comprehensive method, and plays a major role in the cancer treatment. Radiotherapy acts via the induction of Double Strand Breaks (DSBs) into DNA, and then triggers inner cellular self-defense mechanism to induce apoptosis of cancerous cells via programmed cell death [1], [3]. It is important to study the potential genetic and environmental risk factors together in order to understand the mechanisms of underlying aetiology.

The main factor leading to success in clinical radiotherapy is radiation dose. Low doses are ineffective, whereas if it is possible to give a very large radiation dose then, in principle, any tumor can be (locally) controlled, but it will bring unavoidable damage to the normal cells adjacent to the tumor [1], [4]. Currently, some combined approaches of information science, control theory, and system biology are invoking new ideas on the investigation of complicated mechanisms of bio-system at single cell level [5]. Some theoretical methods have been proposed to investigate cellular self-defensive mechanism under genome stress, such as Gene Regulatory Network (GRN) models [6]. In addition, Gene-Environment Network (GEN) model, a novel mathematical framework, has been widely investigated by using the kinetic theory of active particle (KTAP) [7]-[9]. KTAP can model the overall bio-system by evolution equations corresponding to the dynamics of all elements within different subsystems [9]. GEN can describe not only the stochastic interactions between genes and environmental cofactors at molecular level, but also the systematic dynamics of integrated system in response to environmental perturbations [9], [10]. GEN model is widely used in different research 
areas, e.g., cellular self-repair kinetics under acute IR [11], as well as cellular fate decision by gene cofactors [12], [13]. Especially, KTAP has been applied to some areas in social and life sciences, e.g. social behavior of interacting individuals [14], as well as multi-cellular system and tumor-immune system competition [15], [16].

To investigate the simulation of the tumor radiotherapy mechanism further, we propose a hybrid model based on KTAP framework and GEN method. In the following paragraphs, the second part presents the mathematical framework for the mutual interactions among active particles at molecular level, and the dynamic kinetics of cellular response at systematical level. The third part illustrates the dynamic outcomes of the molecular interactions under acute IR cofactors, including the kinetics of stochastic DNA damage generation, DNA repair, and switch-like Ataxia-Telangiectasia-Mutated (ATM) activation; periodical oscillation of P53 network; and alternative phase of cell cycle arrest and apoptosis. The fourth part predicts the outcome of radiotherapy by selecting some indicators, and thus analyzes how to find an optimal therapy strategy by comparing the outcomes of radiotherapy under different IR time in effective IR range.

\section{Methods}

\subsection{Overview of the Hybrid Model}

As shown in Fig. 1, the hybrid model is composed of three subsystems: DNA damage transferring and ATM activation, P53 network oscillation, and tumor cell cycle arrest and apoptosis. In terms of the KTAP framework and GEN model, we deal that all of three subsystems consist of active molecular particles with different microscopic discrete states. Therefore, the mutual interactions between molecular particles and environmental co-factors can be presented at molecular level, which may modify either the state or the number of molecular in each subpopulation by proliferation/ destruction phenomena [12], [16]. Especially, the dynamic kinetics of the hybrid model can be dealt as results of mutual interactions among correlated molecular particles in different subsystems and outer environmental cofactors. In this method, some vital genes and their mutual interactions with environmental cofactors are involved. Due to the complicated mechanisms of radiotherapy, this hybrid model just provides a platform for investigating the simulations of clinical radiotherapy kinetics theoretically. Meanwhile, the detailed mathematical formulations are omitted due to the page limitation.

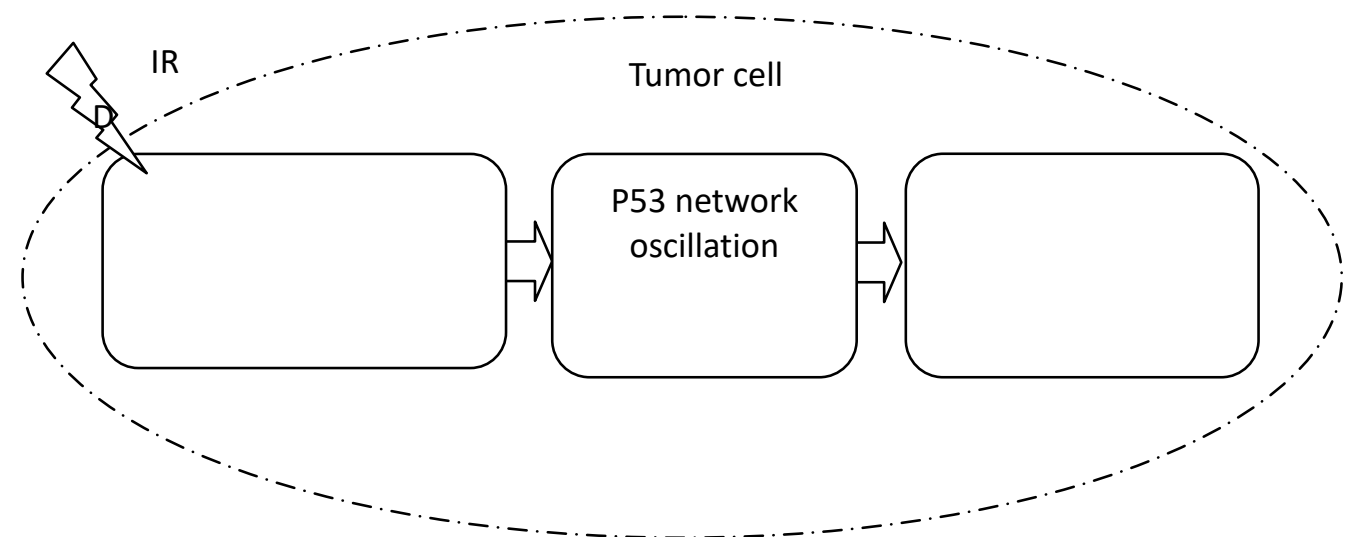

Fig. 1. The scheme of the hybrid model for tumor radiotherapy.

It is composed of three correlated subsystems: DNA damage transferring and ATM activation, P53 network oscillation, and tumor cell arrest \& tumor cell apoptosis. Each subsystem includes active molecular particles of several populations with different discrete states. 


\subsection{DNA Damage Transferring and ATM Activation}

In this model, the first subsystem shown in Fig. 2 tries to present the stochastic kinetics of DNA damage generation, repair and transfer, as well as ATM activation under radiotherapy. As acute IR perturbation is applied into a tumor cell, DSB, a typical form of DNA damage, occurs stochastically. Providing cellular self-repair mechanism is available, some repair enzymes can be quickly recruited to bind into the nascent DNA ends, then the DSB-protein Complexes (DSBCs) are formed [17]. As a sensor of genome stress, ATM kinase is very sensitive for these fixed DSBCs, thereafter, the switch-like kinetics of ATM activation is triggered through intermolecular phosphorylation [18], under mutual interactions with damage signals transferred from DSBCs synthesis. In the stochastic DSBs generation process, we consider the fact that different numbers of DSBs are generated along with different IR dose domains, and then deal that the number of resulting DSBs induced per IR dose within each time scale obeys the principle of Poisson random distribution, whose average is proportional to the radiation dose [1], [16]. Generally, most of the resulting DSBs can be fixed by cellular self-repair mechanism. During DNA damage repair process, the kinetics of RP translation is prompted through mutual interactions of intact DSBs with repair mRNA. For repairable DNA damage during the first phase of radiotherapy, the available RP is quickly recruited to the damage sites, and then synthesize DSBC after combining into the resulting DSB [19]. In this module, the corrected repair part of DSBC (rDSBC) is dealt as main signal source to trigger switch-like ATM activation. However, for un-repairable DNA damage during the second phase of radiotherapy, the intact DSBs remain and increase dramatically in cell, which can seriously weaken genome stability and cellular viability [20]. Therefore, we deal that the total concentration of intact DSBs and mis-repaired part of DSBC (mDSBC) as cellular toxins generated during and after tumor radiotherapy, and as one indicator of the outcomes of tumor radiotherapy [7], [21].

Meantime, in response to positive interaction with DSBC particle transferred from DSB repair process, ATM dimer (ATMd) converts into inactive ATM monomer (ATMm), and then transforms into active ATM monomer $\left(\mathrm{ATM}^{*}\right)$ [20], [21]. In fact, ATM activation is a reversible kinetics, i.e., ATMm can convert into ATMd, and ATM* can transform into ATMm reversibly. Meanwhile, we deal that the total concentration of ATM including ATMd, ATMm and ATM* is constant, because it mainly undergoes posttranslational modifications after DNA damage. In addition, ATM dimers are predominant in unstressed cells, as the dimerization rate of ATM is much larger than its undimerization rate [21].

\subsection{P53 Network Subsystem}

P53-MDM2 negative feedback loop is important to trigger downstream genes, and then regulate a series of cellular mechanism under radiotherapy, such as cell cycle arrest and cell apoptosis etc. As an important tumor suppressor, active P53 ( $\left.\mathrm{P}^{*} 3^{*}\right)$ is very low in unstressed cell. However, to eliminate lethal genome damage or deregulated proliferation, the level of P53* can increase quickly in some minutes upon DNA damage transferring in response to external perturbation cofactors, while the first cellular apoptotic event occurs in a few hours in some cell types [20], [21].

As shown in Fig. 3, we mainly deal the P53-MDM2 negative feedback loop as a kernel part in the P53 network subsystem. Within DNA damage signal transferring from upstream ATM activation, the kinetics of

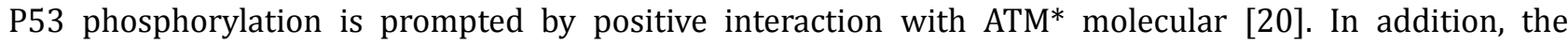
dynamics of MDM2 degradation is accelerated by negative interaction with ATM* particle as well. Meantime, the expression of MDM2 is triggered by positive interaction with P53* molecular further, whereas, the concentration of P53* can be depressed by MDM2, a P53-specific ligase and antagonist of P53, in order to avoid the over-expression of P53 [18]. Thereafter, the dynamic equilibrium of P53-MDM2 feedback loop will be impaired by ATM-dependent phosphorylation of P53 and MDM2. As a result, some periodical oscillations 
occur between P53 and MDM2 as expected [7], [20].

\subsection{Tumor Therapy Subsystem}

P53 and its cofactors, e.g., P21 and Bax are vital for cell fate decision, especially for the outcomes of radiotherapy [13]. In the tumor therapy subsystem, as shown in Fig. 4, we mainly consider the P21-dependent cell cycle arrest and Bax-dependent apoptosis signal pathway. In terms of the extent of DNA damage, the P53-dependent signal pathway can make a reliable decision between survival and cell apoptosis. During the first phase of radiotherapy, the active P53 sustains a moderate level upon repairable DNA damage. Under positive interaction with low level of P53* molecular particles, P21-dependent signal pathway can be initiated directly, and then the damaged cell undergoes a transient cell cycle arrest period. As a result, the kinetics of DNA damage repair is prompted, and then tumor cell proliferation is blocked temporarily [13], [17]. On the other hand, for irreparable DNA damage in the second phase of radiotherapy, Bax-dependent cell apoptosis pathway can be evoked alternatively by positive interaction with high level of P53* particles [5], [17], [22]. Meanwhile, we deal that tumor cell can be degraded directly by Bax-dependent signal pathways, although some other intermediate steps, e.g., CytoC release and Casp3 activation, are also necessary for regulating cell apoptosis mechanism [17], [22].

\section{Impletentation}

To reveal the dynamic kinetics of tumor radiotherapy, a series of cellular activities are illustrated through MATLAB simulation platform, including stochastic kinetics of DNA damage generation, repair and transfer; switch-like ATM activation; oscillations of P53-MDM2 feedback loop; alternative activation of P21, Bax-dependent cell cycle arrest and cell apoptosis signal pathway. Furthermore, the outcomes of tumor radiotherapy are analyzed by introducing some indicators such as, the number of RP available, toxins, tumor cell mass, cellular activity as well. Then, an optimal radiotherapy strategy is detected from different IR perturbation circumstances.

DNA damage transfer and ATM activation. In this simulation, the initial number of resulting DSBs per time scale is dealt as proportional number generated by Poisson random function with a mean of $35 \mathrm{x}$, in which $\mathrm{x}$ is the strength of IR dose [5], [6]. First, we apply IR=3Gy as an example from these radiotherapy strategies above. As shown in Fig. 5, the resulting DSBs occur stochastically under external interactions with constant 3Gy IR cofactor (Fig. 5). Due to fewer DSBs generated under lower IR, the number of synthesized rDSBC keeps increasing with enough RP (Fig. 5) available around DNA damage sites. Meanwhile, the accumulated toxins (Fig. 5) which mainly include mDSBC, increase slowly in a tumor cell. In response to DNA damage transferring from rDSBC, the switch-like ATM is activated from inactive ATM monomer (ATMm) (Fig. 5), and phosphorylated ATM monomer (ATM*) (Fig. 5) rapidly rises to a high plateau after about 300 time-scales, and then keeps a dynamic equilibrium versus continuous radiation time.

Oscillations of P53 network subsystem. The periodic oscillations occurred in P53-MDM2 negative feedback loop are important clues to investigate the inner mechanism of tumor radiotherapy [5], [22]. Within damage signal transferring from switch-like ATM activation, the dynamic pulses between P53 and MDM2 are initiated, and the number of these pulses is mainly determined by the duration of ATM activation process. In this part, we take IR=23Gy as another therapy strategy, and illustrate the dynamic oscillations between P53 and MDM2. As shown in Fig. 6, the saturated P53 mRNA provides continuous signal resource to trigger P53-MDM2 oscillator (Fig. 6). Upon both positive interaction with ATM* and negative interaction with MDM2, P53 is activated quickly from P53, and keeps high level with periodic pulses (Fig. 6). Meanwhile, upon the positive interaction with activated P53 (P53*) and negative interaction with ATM* molecule, MDM2 is translated from MDM2 mRNA, and owns similar periodic pulses with P53* (Fig. 6). These oscillations between P53 and MDM2 have similar periods of about 400 time-scales with different phases 
and swings, and the first pulse is slightly higher than the second one, and then tends to disappeared after about 1400 time-scale (Fig. 6), as ATM* signal is vanished with the limited cellular self-repair capability in response to much more intensive IR dose and longer therapy time.

Outcomes of cell cycle arrest $\&$ apoptosis. In this part, we apply IR=43Gy of radiotherapy into a tumor cell. As shown in Fig. 7, in response to repairable DNA damage during the first phase of radiotherapy, P21 is activated from P21 mRNA, and kept saturation state for about 150 time-scale, after positive interaction with relative low level P53* (Fig. 7). On the contrary, in response to un-repairable DNA damage, Bax is activated from Bax mRNA after positive interaction with relative high level P53*, and then decreases and tends to zero slowly after reaching a concentration climax (Fig. 7). Suppose that tumor cell mass can be directly degraded by Bax-dependent signal pathway, under continuous radiation time of 43Gy therapy strategy, tumor cell mass keeps increasing slowly and tends to equilibrium constant (Fig. 7); meanwhile, the activity of tumor cell keeps decreasing and tends to zero, because more toxins are accumulated in the cell. These simulations above denote that the outcome of radiotherapy under IR=43Gy circumstance is not encouraging, because a lot of accumulated toxins can seriously decrease genome stability and cell activity, especially, the tumor cell cannot be degraded efficiently during the second phase of this therapy strategy.

Moreover, the results of cell cycle arrest and apoptosis kinetics under different IR circumstances are listed in Table 4, we can see that, as IR gets bigger, the maximal value of P21 keeps decreasing, whereas, the maximal value of Bax keeps increasing, and then sharply decreases after IR overtakes 33Gy. Especially, under IR=33Gy, both the minimal and maximal value of tumor cell mass gets the smallest one in all six IR strategies. These analyses suggest that an optimal therapy strategy is available near 33Gy IR, in which tumor cell can be eliminated efficiently. Otherwise, the outcomes of radiotherapy are not encouraging if the strength of IR is too low or too high, because the mechanism of cell apoptosis cannot be initiated during the second phase of radiotherapy.

Optimal strategy for tumor therapy. Generally, the radiation time and the strength of IR dose are important for outcomes of radiotherapy. In this part, we take some primary elements from the integrated OFTR model, and then roughly analyze the results of radiotherapy under different therapy circumstances, in order to find an optimal strategy with smaller tumor cell mass and fewer toxins remained, as well as stronger cell activity during and after radiotherapy.

In our simulations, we apply different IR range from 24 to $42 \mathrm{~Gy}$ into a tumor cell, respectively. The selected and summarized results are illustrated in Fig. 8. For IR=28, 32, 36, and 40Gy, as shown in Fig. 8, the period of ATM activation and P53 network oscillation gets shorter when IR becomes stronger. Meanwhile, the first phase of radiotherapy, i.e., cell cycle arrest triggered by P21-dependent signal pathway, becomes shorter and weaker; on the contrary, the second phase of therapy, i.e., cell apoptosis induced by Bax-dependent signal pathway, gets longer and stronger, and dominates the main therapy process. As a result, tumor cell mass can be degraded more efficiently during the second phase of radiotherapy. However, due to the increasing toxins remained, the activity of tumor cell keeps decreasing versus continuous therapy time under different IR strategies.

\section{Conclusions}

In this work, the hybrid model for radiotherapy with three subsystems is proposed by using KTAP framework and GEN. Our method can successfully exhibit a series of dynamic kinetics of tumor radiotherapy from molecular to systematic levels, including the stochastic process of DSB generation and repair, switch-like ATM activation, periodic oscillations of P53-MDM2 negative feedback loop, alternative kinetics of both P21-dependent cell cycle arrest and Bax-dependent cell apoptosis signal pathways. The simulation results show the proposed model can be effectively used to explore the inner mechanism of 
tumor radiotherapy from molecular to systematic level, and then further provide some useful clues and advices on designing more efficient radiotherapy strategy in theory.

\section{Acknowledgment}

This paper is supported by National Natural Science Foundation of China (no. 13K10414, no. 61305081 and no. 61104154), Specialized Research Fund for Natural Science Foundation of Shanghai (no. 16ZR1401300 and no.16ZR1401200) and Shanghai Postdoctoral Sustentation Fund (no. 12R21410200).

\section{References}

[1] Perez, C., \& Brady, L. (1999). Principles and practice of radiation oncology. Journal of Pediatric Hematology/Oncology, 21, 560.

[2] Amato, R., Pinelli, M., D'Andrea, D., Miele, G., Nicodemi, M., Raiconi, G., et al. (2010). A novel approach to simulate gene-environment interactions in complex diseases. BMC Bioinformatics, 11, 8.

[3] Magné, N., Toillon, R, A., Bottero, V., et al. (2006). NF- $\mathrm{B}$ modulation and ionizing radiation: Mechanisms and future directions for cancer treatment. Cancer Letters, 231, 158-168.

[4] Bellouquid, A., \& Delitala, M. (2006). Modelling Complex Multicellular Systems-A Kinetic Theory Approach. Birkäuser, Boston.

[5] Ma, L., Wagner, J., Rice, J, J., Hu, W., Levine, A. J., \& Stolovitzky, G, A. (2005). A plausible model for the digital response of p53 to DNA damage. Proceedings of the National Academy of Sciences of the United States of America (vol. 102, pp. 14266-14271).

[6] Qi, J. P., Shao, S. H., Li, D. D., \& Zhou, G, P. (2007). A dynamic model for the p53 stress response networks under ion radiation. Amino Acids, 33, 75-83.

[7] Hahn, L. W., Ritchie, M. D., \& Moore, J. H. (2003). Multifactor dimensionality reduction software for detecting gene-gene and gene-environment interactions. Bioinformatics, 19, 376-382.

[8] Bellouquid, A., \& Delitala, M. (2006). Mathematical modeling of complex biological systems: A kinetic theory approach. Springer.

[9] Brazzoli, I. (2008). From the discrete kinetic theory to modelling open systems of active particles. Applied Mathematics Letters, 21, 155-160.

[10] Angelis, E. D., \& Lods, B. (2008). On the kinetic theory for active particles: A model for tumor-immune system competition. Mathematical and Computer Modelling, 47, 196-209.

[11] Qi, J. P., Ding, Y. S., Zhu, Y., \& Wu, Y. Z. (2011). Kinetic theory approach to modeling of cellular repair mechanisms under genome stress. PloS one, 6, e22228.

[12] Bellomo, N. (2008). Modeling complex living systems: A kinetic theory and stochastic game approach. Springer.

[13] Zhang, X. P., Liu, F., \& Wang, W. (2012). Regulation of the DNA damage response by p53 cofactors. Biophysical Journal, 102, 2251-2260.

[14] Bertotti, M, L., \& Delitala, M. (2008). Conservation laws and asymptotic behavior of a model of social dynamics. Nonlinear Analysis: Real World Applications, 9, 183-196.

[15] Bellomo, N., and Forni, G. (2008). Complex multicellular systems and immune competition: New paradigms looking for a mathematical theory. Current Topics in Developmental Biology, 81, 485-502.

[16] Li, L., Story, M., \& Legerski, R. J. (2001). Cellular responses to ionizing radiation damage. International Journal of Radiation Oncology Biology Physics, 49, 1157-1162.

[17] Pauklin, S., Kristjuhan, A., Maimets, T., \& Jaks, V. (2005). ARF and ATM/ATR cooperate in p53-mediated apoptosis upon oncogenic stress. Biochemical and Biophysical Research Communications, 334, 386-394.

[18] Ritter, M. A. Gilchrist, K., Voytovich, W. M., Chappell, R. J., \& Verhoven, B. M. (2002). The role of p53 in 
radiation therapy outcomes for favorable-to-intermediate-risk prostate cancer. International Journal of Radiation Oncology Biology Physics, 53, 574-580.

[19] Budman, J., \& Chu, G. (2005). Processing of DNA for nonhomologous end - joining by cell - free extract. The EMBO Journal, 24, 849-860.

[20] Kohn, K, W., \& Pommier, Y. (2005). Molecular interaction map of the p53 and Mdm2 logic elements, which control the Off-On switch of p53 in response to DNA damage. Biochemical and Biophysical Research Communications, 331, 816-827.

[21] Tribius, S., Pidel, A., \& Casper, D. (2001). ATM protein expression correlates with radioresistance in primary glioblastoma cells in culture. International Journal of Radiation Oncology Biology Physics, 50, 511-523.

[22] Vogelstein, B., Lane, D., \& Levine, A, J. (2000). Surfing the p53 network. Nature, 408, 307-310.

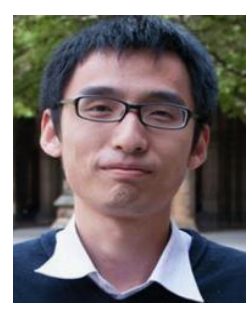

Quan Gu obtained a B.Sc. and M.Sc. in electronic \& electrical engineering and mathematical modelling, and a Ph.D. in bioinformatics at Donghua University, China, in 2002, 2006, and 2011, respectively. He was trained in genomics at the Commonwealth Scientific and Industrial Research Organization (CSIRO, Australia), and previously worked as research associate at Imperial College London. He is currently working as postdoc scientist in the College of Information Sciences and Technology, Donghua University. His research interests include machine learning and pattern recognition, bioinformatics, and Nuclear magnetic resonance (NMR) and mass spectrum (MS) data modelling and analysis. 